\title{
Source Of The Multifractality In Exchange Markets: Multifractal Detrended Fluctuations Analysis
}

Samet Günay, Istanbul Arel University, Turkey

\begin{abstract}
In this study, we analyzed the multifractality and the source of multifractality of the returns of $G B P / U S D, E U R / U S D, U S D / J P Y$ and USD/CHF currencies. In the examination of multifractality we performed the Multifractal Detrended Fluctuation Analysis (MF-DFA). Also, we used shuffled and surrogated data that was derived from the Statically Transformed Autoregressive Process (STAP) method to determine the source of multifractality. According to the results, GBP/USD returns have monofractal features, whereas EUR/USD, USD/JPY and USD/CHF returns have multifractal behaviors. The tests concerning the source of multifractality indicated that the reason of multifractality for EUR/USD and USD/JPY returns is fat-tails of the probability density function of returns, whereas the reason of multifractality of USD/CHF returns are both long memory and fat tails. Also we have seen that there is an ambiguous relationship between the liquidity of the currency market and multifractality.
\end{abstract}

Keywords: Multifractality, MF-DFA, Generalized Hurst Exponent, Multifractal Spectrum

\section{INTRODUCTION}

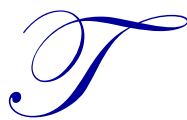

he movements in currency rates and the structure of these movements have important effects ranging from individuals to firms and countries. We can see that there is variety of currency regime experiences in different countries in the historical perspective. Every system has some advantages and disadvantages as usual. For instance in the floating rate regime, although prices are formed in the market depending upon supply and demand equilibrium, sharp and extreme movements can cause large losses in both sides. Hence, some methods and financial instruments have been developed in order to manage these types of risks. However, the most important point in this issue is not the variety of the techniques; the question is which methods properly define the real structure of these assets' price process? Beyond the currency regimes, currency rate risks and risk management instruments; in this study we will analyze the answer to this question for four important currency markets and try to define the new generation "stylized facts" of the currency markets via Multifractal Detrended Fluctuations Analysis.

In order to analyze the financial time series' idiosyncratic features, many studies have been conducted in conjunction with the availability of high frequency data in financial markets. As an output of these studies, we have seen that there are some "stylized facts" in the financial time series, such as fat tails and volatility clustering (Segnon and Lux, 2013). In the last 50 years, Mandelbrot's brushstrokes with fractality concept have caused irreversible, significant changes to the conventional finance theory based on the random walk. Therefore, Mandelbrot added new realities to the existing "stylized facts", such as long memory and self-similarity. The assumption that the financial asset returns do not have correlation under the Efficient Market Hypothesis has been criticized severely after the studies of Mandelbrot $(1963,1966,2004)$, and studies have explicitly shown that nonlinear return functions (squared and absolute returns) have long memory properties (Pochart and Bouchaud, 2002).

The fractality concept that is based on the assumption that the financial times series may have long range dependence that consists of scaling and power law notions. Scaling defines the relationships of the returns in 
different time scales such as daily, weekly and monthly (Mandelbrot et al., 1997a). The occurrence of extreme events in the returns of the financial time series creates fat tails in the return distributions. Due to these fat tails that are derived from large returns, scaling functions decay hyperbolically, instead of exponentially. In other words, the time series that have fractality features scale in accordance with the power law. In the literature, the first definition in exhibiting scaling properties of financial time series is Mandelbrot's (1972) Hurst exponent obtained via $R / S$ analysis. If the $x(t)$ random walk process is self similar, it satisfies the scaling rule below:

$\chi(c t)=\chi(t) c^{H}$

where $t$ is the time scale, $c$ is a constant, and finally, $H$ denotes the Hurst exponent. When we increase the time increment, the Hurst exponent, $0.5<H<1$, demonstrates that scaling occurs in accordance with a the power law; that is, the $x(c t)$ value is scaled with the power law that is equal to $H$ (Peters, 1994). The Hurst exponent, $H$, in Equation (1) is constant, and the relation variates follow the nonlinear power law defined by $H$.

In recent studies, Mandelbrot (1997) has shown that the time series may have more than one scaling exponent. In such circumstances, multifractal analysis that uses multiple scaling exponents provides more confidential results than one scaled exponent monofractal analysis. As it was explained by Kantelhardt et al. (2002) many time series do not exhibit monofractal scaling behaviours. Under these conditions, using only one scaling exponent does not provide enough to define the characteristics of the time series because different regimes of the series may have different scaling values. In such cases, in order to define the scaling behaviour properly, we need multiple scaling exponents as seen in the multifractal model below:

$\chi(c t) \equiv \chi(t) c^{h(q)}$

where $h(q)$ denotes the generalized Hurst exponent that is unconstant and different from the Hurst exponent in Equation (1). It means that, under different $q$ orders, $h(q)$ can have different values. A constant $h(q)$ means monofractality and $h(2)$ is equal to the classical Hurst exponent. Multifractality is a scaling property that considers both extreme events and long memory. Therefore, in a multifractal analysis both fat tails, in the unconditional distributions of return, and long memory, in the absolute returns, are taken into account (Calvet and Fisher, 2002).

\section{LITERATURE REVIEWS}

Mandelbrot's (1972) Rescaled Range analysis $(R / S)$, that is based on the study of Hurst (1951), has been used by many researchers in the testing of the scaling behaviours of the financial time series in future years. Peng et al. (1994) performed a different way called the Detrended Fluctuation Analysis (DFA) in the computing of the Hurst exponent, and provided a robust alternative to the classical $R / S$ analysis.

In spite of the fact that the fractal split in two as monofractals and multifractals, until the studies of Mandelbrot et al. (1997a,b), previous studies were centered generally on monofractal structures. Mandelbrot et al. (1997a,b) presented a new model, known as the Multifractal Model of Asset Returns (henceforth, MMAR), as an alternative to the ARCH type models. The key features of this model take long memory and fat tails in the return distribution into account. For instance, Fillol (2003) showed the outperforming of MMAR against the GARCH and FIGARCH models in his study that analyzed multifractal features of the French Stock Market (CAC40).

In the period following these studies, as an extended version of the Peng et al. (1994) model, Kantelhardt et al. (2002) presented the Multi Fractal Detrended Fluctuation Analysis (henceforth, MF-DFA) that considered the multifractal structure of the unstationary time series. Using the shuffled time series, they also suggested a procedure that determined the source of multifractality. Since then, this model has been used in different fields, from astronomy, to data traffic, to financial time series, and provided successful results in the modeling of multifractality. In one of these studies, Norouzzadeh and Rahmani (2006) tested the Iranian Rial's multifractal structure and scaling properties against the U.S. Dollar. In a different study, Jiang and Zhou (2008) performed MF-DFA to analyze multifractal features of two index volatilities from the China stock market, and regarding the idea of ensemble, reported that China stock markets have multifractal properties. Likewise, Yuan et al. (2009) exhibited the existence of multifractality in the Shanghai stock market, and stated that the reason of multifractality is fat-tailed probability 
distributions and non-linear temporal correlations. In spite of that, Du and Ning (2008) reported weak multifractality for the Shanghai stock market. In a different study for the China stock market, Wang et al. (2009) analyzed the efficiency of the Shenzhen stock market via MF-DFA. The authors split the index into two periods and showed that the index has been gradually more efficient. Another finding is that traditional models such as GARCH and EGARCH are not successful in the forecasting of volatilities. On the other hand, Zhang (2011) stated that although multifractal spectrums are different in both China and Japan's stock markets, they also showed that China's stock market has larger and more frequent fluctuations. For Asian stock markets more recently, Niere (2013) examined the multifractality of the Peso/Dollar currency rate and reported that source of the multifractality is fat-tail in the probability distributions. Similar to Wang et al. (2009), Lye and Hooy (2012) tested the efficiency of the Malaysian stock market. According to their findings, multifractality in the Malaysian stock market arises from the long memory and fat tails; the Malaysian sectoral efficiency has been impacted negatively by Asian and global financial crises. In a study from India Kumar and Deo (2009) showed the multifractality of the BSE \& NSE indexes.

In the studies from European stock markets, there is also evidence of multifractality. For example, Caraiani (2012) used the EMB-based MF-DFA analysis to test the multifractal structure of Czech, Hungarian and Polish stock markets. According to the results, multifractal spectrums and variations of the generalized Hurst exponent for different $q$ orders provided signs of multifractality for the three stock markets. Pleşoianua et al. (2012) analyzed the Romanian stock markets' informational efficiency via one dimensional backward multifractal detrended moving average (MF-DMA) method, and investigated the source of multifractality with surrogated and shuffled time series. Similar to this study, Ioan et al. (2012) examined multifractal features of the east European countries' (Czech koruna, Croatian kuna, Hungarian forint, Polish zlot, Romanian leu and Russian rouble) currencies against the euro. Results showed that the Russian foreign currency market is the most efficient market within these countries, whereas the Hungarian market's efficiency level is the lowest. Benbachir and El Alaoui (2011) investigated the source of multifractality in the Moroccan All Shared Index (MASI) and the Moroccan Most Active Shares Index (MADEX) using shuffling and the phase randomization techniques. Their findings showed that the MASI index has a higher level of multifractality than the MADEX index. Also they reported that similar to the findings of Lye and Hooy (2012), the reason of fractality in the MASI and MADEX indexes are long memory and fat tails in the probability distribution. Liu et al. (2008) tested multifractality of different assets such as stocks, currencies and bonds using the Markov-switching multifractal model. According to the findings of their analysis, the Lognormal Markov-switching multifractal model and Binomial Markov-switching multifractal model analysis gave approximately the same results. Qian et al. (2011) improved a new MF-DFA model based on the empirical mode decomposition, named EMD-based MF-DFA. The authors showed that when the moment order $\mathrm{q}$ of the detrended fluctuations is positive, EMD-based MF-DFA outperforms classical MF-DFA.

\section{METHODOLOGY: MULTIFRACTAL MODEL AND MULTIFRACTAL DETRENDED FLUCTUATIONS ANALYSIS}

Using the definition of Mandelbrot et al. (1997b), we can exhibit the scaling law in multifractality as follows: Let $X(t, \Delta t)$ denote increments of the sthocastic process $X(t)$. In this case:

$X(t, \Delta t) \equiv X(t+\Delta t)-X(t) \quad 0 \leq t \leq T$

Mandelbrot et al. (1997b) defines a multifractal process with stationary increments and it satisfies the equation below:

$\mathbb{E}\left[|X(t, \Delta t)|^{q}\right]=c(q)(\Delta t)^{\tau(q)+1}$

where for all $t$ and q, $\mathbb{E}\left[|X(t, \Delta t)|^{q}\right]<\infty$. Equation (4) is the scaling law in the moments of $X(\Delta t)$ and it specifies a form for their change as $\Delta t$ varies. All information about the growth rate is within the scaling function $\tau(q)$. Therefore $\tau(q)$ is the focal concept within the multifractal theory. Kantelhardt et al. (2002) adapted the DFA model of Peng et al. (1994) to the multifractal concept with reference to this definition. In order to define MF-DFA, let's examine the $x_{k}$ series. $x_{k}=0$ can be interpreted as there is not any value in the related $k$. The first three steps of the MF-DFA is identical with the classical DFA. MF-DFA consists of five steps: 
Step 1. In the first step the profile $Y(i)$ is determined

$Y(i) \equiv \sum_{k=1}^{i}\left[x_{k}-\bar{x}\right], \quad i=1, \ldots, N$

Step 2. Then $Y(i)$ is split into $N_{s}$ sub-segments. The same procedure is also done again starting from the other side of the series, and hence the $2 N_{s}$ segment is obtained.

Step 3. After local trends are determined by OLS for every segment, variance function is defined as follows

$F^{2}(v, s) \equiv \frac{1}{s} \sum_{i=1}^{s}\left\{Y[(v-1) s+i]-y_{v}(i)\right\}^{2}$

where $y_{v}(i)$ is the fitting polynomial in every segment $v$. These polynomials can be linear (DFA1), quadratic (DFA2), cubic (DFA3) or higher degrees.

Step 4. In order to obtain the q order fluctuation function, all of the segments are averaged,

$F_{q}(s) \equiv\left\{\frac{1}{2 N_{s}} \sum_{v=1}^{2 N_{s}}\left[F^{2}(v, s)\right]^{\frac{q}{2}}\right\}^{\frac{1}{q}}$

where $F_{q}(s)$ will increase with the rise of $s$

Step 5. In the last step, scaling behaviour of fluctuation functions are determined by plotting log-log graphs of $F_{q}(s)$ versus $s$ for every value of q. In the case that there is long term power law correlation in the series, we obtain the result below

$F_{q}(s) \sim s^{h(q)}$

As it is stated, for the stationary series $h(2)$ will be equal to . Therefore the $h(q)$ function is called the generalized Hurst exponent. $h(q)$ has a close relationship with the classical multifractal scaling exponent $\tau(q)$. This relationship can be exhibited as follows

$\tau(q)=q h(q)-1$

Another way of characterizing multifractal time series is the singularity spectrum $f(\alpha)$. Connection of the $f(\alpha)$ and $\mathrm{h}(\mathrm{q})$ can be demonstrated in the following way:

$f(\alpha)=q \alpha-\tau(q)$

where $\alpha$ is the singularity strength or Hölder exponent.

\section{EMPIRICAL ANALYSIS}

In this section of the study, we investigate the multifractal structure of the returns of different currency markets via MF-DFA, and in the case of seeing any multifractality signs on the returns, we examine the source of multifractality. All the data used in empirical analysis obtained via the Bloomberg data base and consist of daily frequency. As we do not want to let the liquidity effect on the results of analysis, we preferred to use the most liquid currency markets presented in the Bank for International Settlements' (BIST) reports. These currency markets are the US Dollar, the Japanese Yen, the British Pound, the Swiss Franc and the Euro, and the currency pairs are the 
British Pound/US Dollar, the Euro/US Dollar, the US Dollar/Japanese Yen and the US Dollar/Swiss Franc (henceforth; GBP/USD, EUR/USD, USD/JPY and USD/CHF, respectively). The time interval of the series is from $01 / 01 / 2004$ to $02 / 25 / 2014$, and the returns of the currencies are calculated in the following way:

$R_{c}=\log \left(\frac{P_{t}}{P_{t-1}}\right)$

where, $P$ is price in time, $t$, and $R_{c}$ is the logarithmic returns of the related currency.

\section{1. Multifractal Detrended Fluctuations Analysis}

Before we start the empirical analyses, in the first instance, we followed the first three steps suggested by Kantelhardt et al. (2002), and using Equation (7), we calculated the fluctuation functions $F_{q}$ (s) for 101 different $q$ orders. Multiscaling behaviours of the $F_{q}(\mathrm{~s})$ versus the time scale $s$ are presented in Figure 1 below. Figure 2 demonstrates the similar behaviours for fitted lines obtained via the ordinary least squares method with three different $q$ values $(q=-5, q=0$ and $q=5$ ).
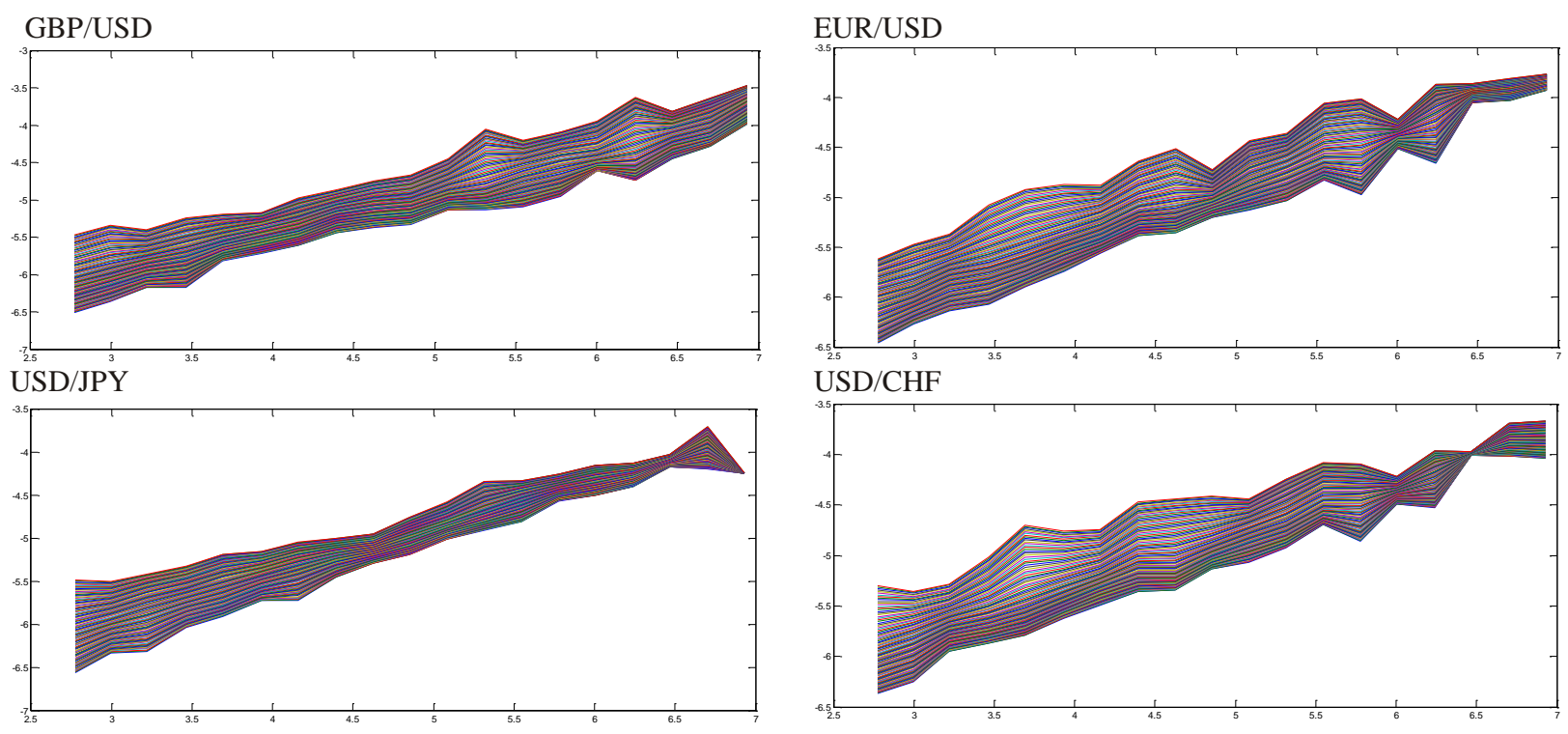

Figure 1. The plotting of $\log F_{q}(\mathrm{~s})$ vs. $\log s$ of the series

As it can be seen from both groups of plots, the fluctuation functions of the GBP/USD returns are almost a parallel in every scale level, whereas the functions of the EUR/USD, USD/JPY, USD/CHF returns approach each other at high scale values. In other words, the slope of the fluctuation functions of the GBP/USD returns are approximately constant. In this stage these findings can be interpreted as monofractal behaviours of the GBP/USD returns. As it has been mentioned earlier, in the monofractality situation, we have only one generalized Hurst exponent value $h(q)$ for different $q$ orders. 

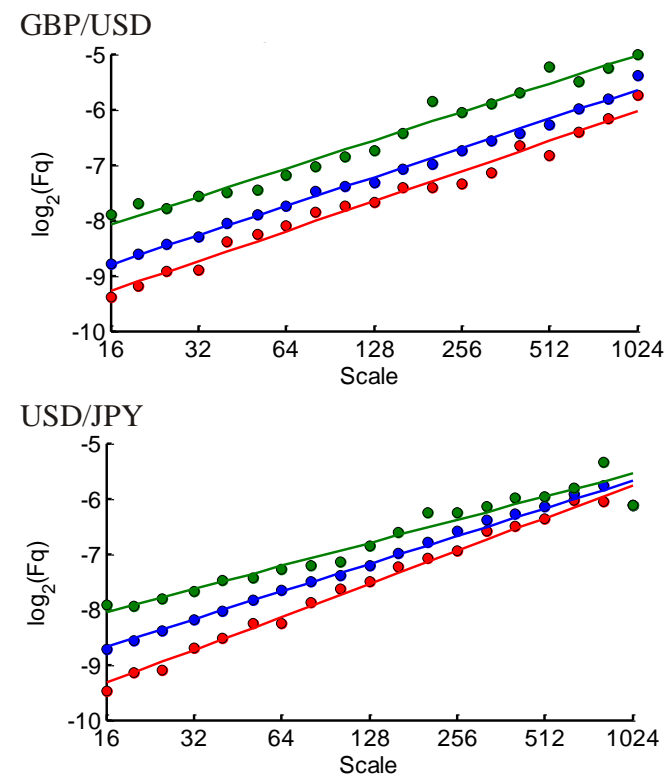
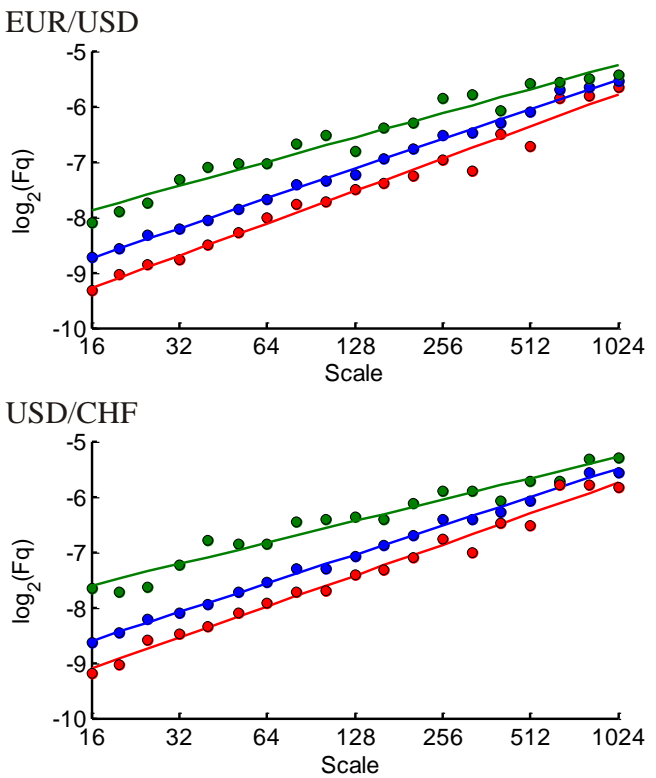

Figure 2. The plotting of $\log F_{q}(s)$ vs. Scale of the series

Table 1 presents the obtained generalized Hurst exponent values $h(q)$ for three different $q$ orders $(-5,0,5)$ that are used throughout the study. One of the important signs of multifractality is the distance of generalized Hurst exponent values from each other for different $q$ orders. According to the results, in accordance with the increase of $q$ orders, obtained $\mathrm{h}(\mathrm{q})$ values decrease. As it is seen, while the highest decrease $(0.1772)$ is in the USD/JPY returns, the lowest decrease is in the GBP/USD returns (0.0322). These findings are related to the path that the generalized Hurst exponent $\mathrm{h}(\mathrm{q})$ function follows versus the increasing $\mathrm{q}$ orders. As a result, the lower the slope of the $\mathrm{h}(\mathrm{q})$ function, the closer the values are to the generalized Hurst exponents. This situation can be seen in Figure 3.

Table 1. q Orders of the Generalized Hurst Exponents

\begin{tabular}{llll}
\hline \hline & $\mathrm{h}(\mathrm{q}=-5)$ & $\mathrm{h}(\mathrm{q}=0)$ & $\mathrm{h}(\mathrm{q}=5)$ \\
\hline \hline GBP/USD & 0.5421 & 0.5263 & 0.5099 \\
EUR/USD & 0.5830 & 0.5380 & 0.4379 \\
USD/JPY & 0.5949 & 0.4983 & 0.4177 \\
USD/CHF & 0.5583 & 0.5196 & 0.3874 \\
\hline \hline
\end{tabular}

Figure 3 demonstrates the path that is followed by the generalized Hurst exponent $h(q)$ of the currency returns versus $\mathrm{q}$ orders. In other words, in this plot we see the dependence of generalized Hurst exponent $\mathrm{h}(\mathrm{q})$ to $\mathrm{q}$ orders. If we look closely, it is seen that, except for the GBP/USD returns, the generalized Hurst exponent $h(q)$ functions of other currency returns have nonlinear decreasing functions, versus the increasing values of q orders. Slopes that are approximately zero give evidence that the time series has a monofractal structure. This circumstance can be seen in the GBP/USD return series clearly. In comparison with other return series, it is obvious that these results arise from the monofractal structure of the GBP/USD returns that have a lower slope in the $\mathrm{h}(\mathrm{q})$ function. 


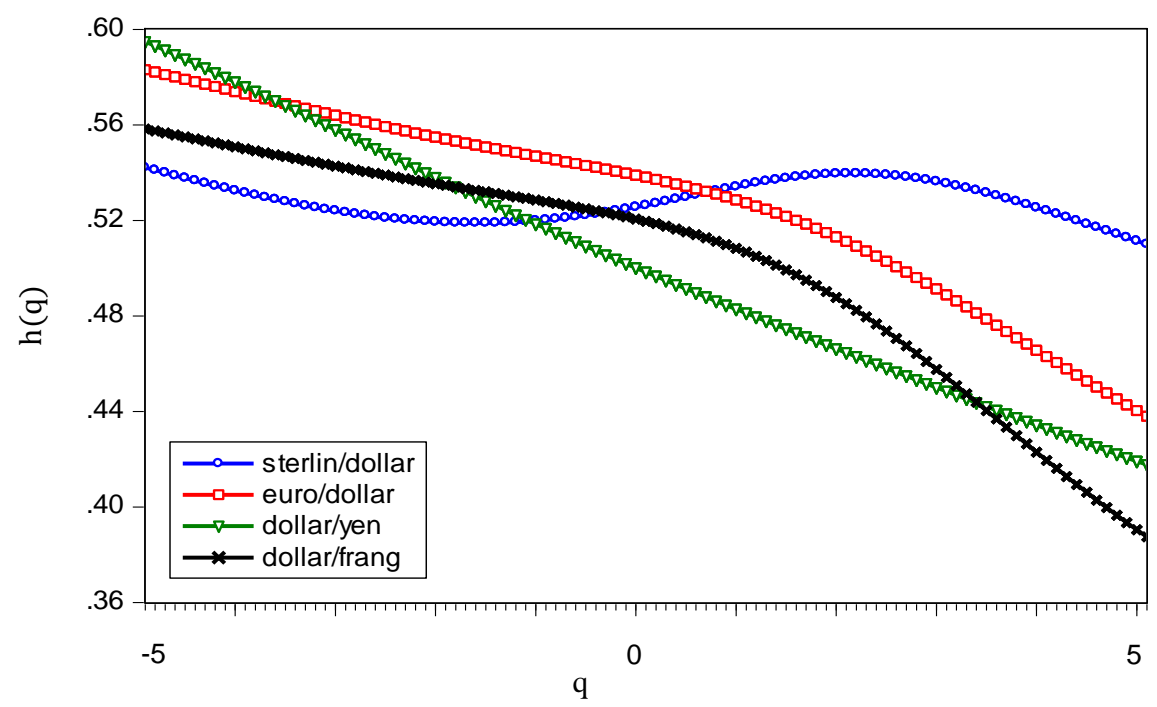

Figure 3. The generalized Hurst exponent h(q) vs. q

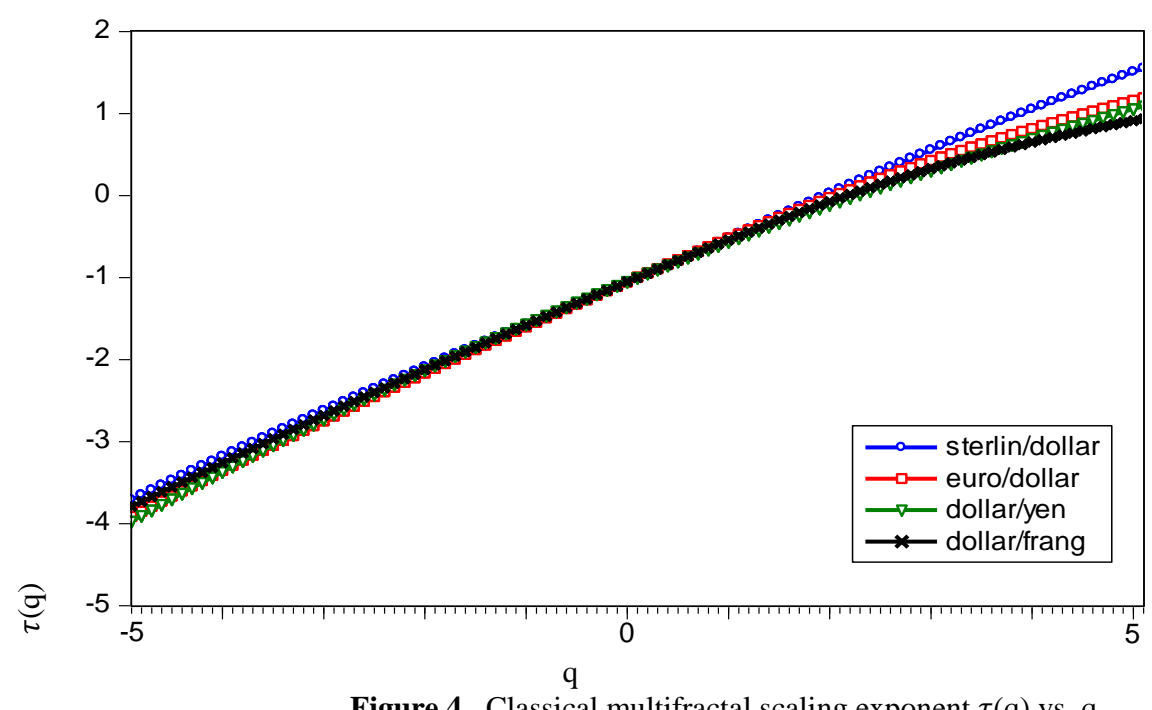

Figure 4. Classical multifractal scaling exponent $\tau(\mathrm{q})$ vs. q

Similar interpretations can be made by examining the plot of the classical multifractal scaling exponent (Reni exponent) versus q orders. As stated by Mandelbrot et al. (1997a), nonlinear relations on this plot demonstrate the existince of multifractal behaviours. Figure 4 exhibits that the $\tau(\mathrm{q})$ values of the EUR/USD, USD/JPY and USD/CHF returns are dependent to q orders nonlinearly. However, the GBP/USD returns perform a linear relationship rather than nonlinear behaviours. Linear and white noise relationships are the sign of monofractality. Consequently, we can say that for the $q<0, q=0$ ve $q>0$ values classical multifractal scaling exponent's $q$ dependency indicate almost identical behaviours in the GBP/USD returns.

As it is stated by Ausloos (2012), in conjunction with the acquiring of the $\mathrm{h}(\mathrm{q})$ function, we can obtain the multifractal spectrum $f(\alpha)$. Where $f(\alpha)$ is the distribution of the exponent $\alpha(\equiv d / d q[q h(q)])$ of the object. The multifractal spectrum is one of the most important tools to provide information about multifractality. The width and shape of the multifractal spectrum indicate the intensity of multifractality and also give information about the sensitivity of multifractality to small and large fluctuations. Deviations from the mean and fractal structure for different segments with small and large fluctuations is presented by the multifractal spectrum width (Ihlen, 2012). 
Table 2 shows the multifractal spectrum statistics for different $q$ orders $(q=-5, q=0$ and $q=5)$ and width values.

Table2. Multifractal Spectrum Statistics and Width Values

\begin{tabular}{llllllll}
\hline \hline & \multicolumn{2}{c}{$\mathrm{q}=-5$} & \multicolumn{2}{c}{$\mathrm{q}=0$} & \multicolumn{2}{c}{$\mathrm{q}=5$} \\
\hline \hline GBP/USD & $\mathrm{f}(\alpha)$ & $\alpha$ & $\mathrm{f}(\alpha)$ & $\alpha$ & $\mathrm{f}(\alpha)$ & $\alpha$ & $\mathrm{h}(\mathrm{q})_{\max }-\mathrm{h}(\mathrm{q})_{\min }$ \\
EUR/USD & 0.7259 & 0.5970 & 1 & 0.5271 & 0.6512 & 0.4401 & 0.1568 \\
USD/JPY & 0.7544 & 0.6322 & 1 & 0.5371 & 0.4168 & 0.3213 & 0.3108 \\
USD/CHF & 0.5539 & 0.6841 & 1 & 0.4966 & 0.6454 & 0.3467 & 0.3374 \\
\hline \hline
\end{tabular}

Benbachir and El Alaoui (2011) reported that the monofractal time series has a multifractal spectrum shape that is dense around the single point where $\alpha=H$ with $f(\alpha)=1$. This form clearly can be seen in the plot of GBP/USD shown in Figure 5. This figure robustly supports the previous results, and it means that the GBP/USD returns have monofractal features instead of multifractal. In the other three plots, EUR/USD, USD/JPY and USD/CHF, unlike the GBP/USD returns' multifractal spectrum shape, the curves have a single-humped shape. That is, the generalized Hurst exponent $\mathrm{h}(\mathrm{q})$ of these three currency returns variate more under different scales, and these currencies have multifractality behaviours.

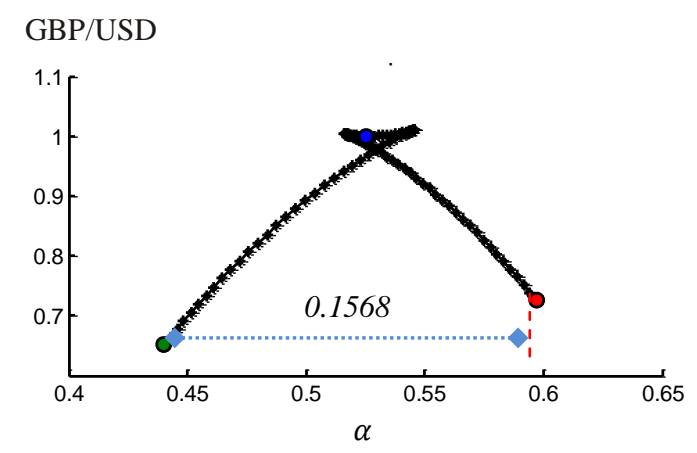

USD/JPY

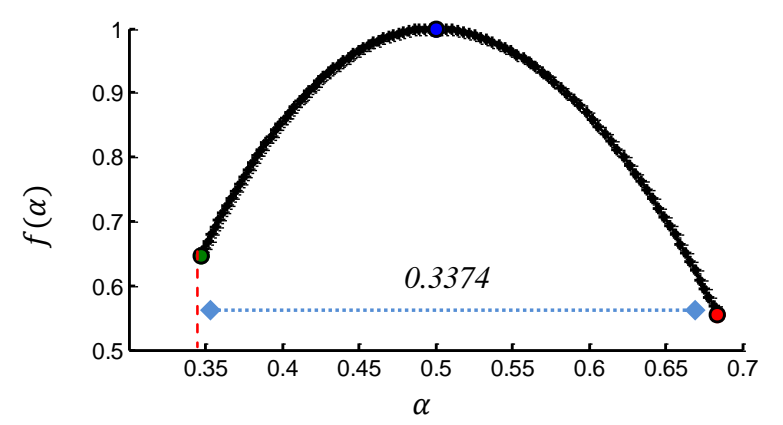

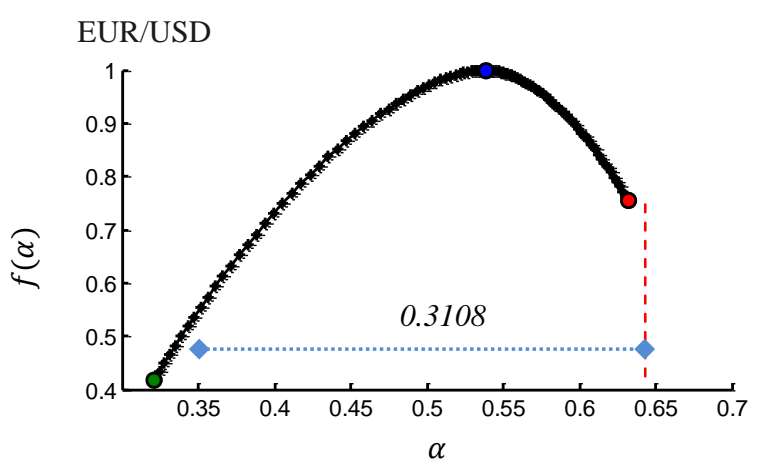

$\mathrm{USD} / \mathrm{CHF}$

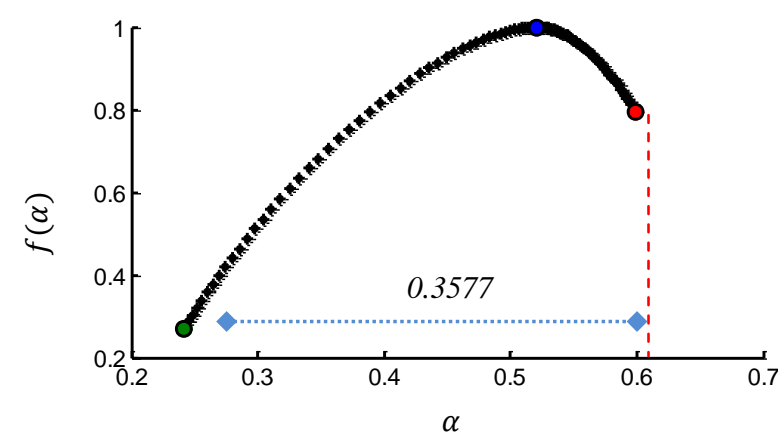

Figure 5. Multifractal Spectrums

As it is stated before, fractal spectrum width gives information about monofractality and multifractality. When the multifractal spectrum width becomes larger, structural differences between the periods with small and large fluctuations will growth. Figure 5 demonstrates that the width for the EUR/USD, USD/JPY and USD/CHF returns are more than 0.30, whereas the multifractal spectrum width of GBP/USD is around only half of that value. This result confirms that unlike the other three currencies, the GBP/USD returns have monofractal behaviours, as well.

\section{2. Source of the Multifractality}

Results up to now showed that, except for the GBP/USD, three of the four currency returns have multifractality features. In this stage of the paper we analyze the source of multifractality in the returns of the 
EUR/USD, USD/JPY and USD/CHF currency rates. Kantelhardt et al. (2002) stated that distinguishing the two different types of multifractality in the time series is possible. The first type of multifractality arises from the fat-tail probability density function. As for the second type, it stems from different long memory features in the small and large fluctuations. In this study, we have used two different types of data in order to reveal the source of multifractality in the EUR/USD, USD/JPY and USD/CHF return series: shuffled data and surrogated time series. As the correlations are non-existent in the shuffled data, the second type of multifractality can easily be identified, whereas the first type cannot be removed by the shuffling procedure due to it arising from the frequency of observations. As the shuffled series will exhibit random behaviours, $h_{s h u f}(q)$ will be equal to 0.5 . In case multifractality arises from the fat-tail in the probability density function, the situation of $h(q)$ in the original series does not change, and $h_{\text {shuf }}(q)$ becomes equal to $h(q)$. Nevertheless, if there is a multifractality that stems from both types, then the shuffled series will exhibit lower multifactality than the original series.

Regarding the first type, we have used surrogated data (phase-randomization techniques) in order to determine the multifractality that arises from the fat-tail in the probability density function following the study of Movahed et al. (2006). Although there are different types of methods in obtaining surrogated data, we preferred the Statically Transformed Autoregressive Process (henceforth, STAP) method because of its flexibility and fiducial inference. Using the surrogate method, we changed the phase of discrete fourier transform (DFT) coefficients with a set of pseudo independent distributed uniform quantities. Therefore, we have transformed the probability density function to the Gaussian distribution without any change in the correlations. Hence, if there is multifractality related with fat-tails, the surrogated series' generalized Hurst exponent $h_{\text {sur }}(q)$ will be independent from the q orders. With the following section we start to analyze the source of multifractality that arises from the long memory features of the series. As it is mentioned before, when the autocorrelations, which stem from the long memory, are removed by the shuffling procedure in the new series, it is expected that $h_{\text {shuf }}(q)=0.5$. On the other hand, if the reason of multifractality is both long memory and fat-tails, the shuffled series has lower multifractality features.

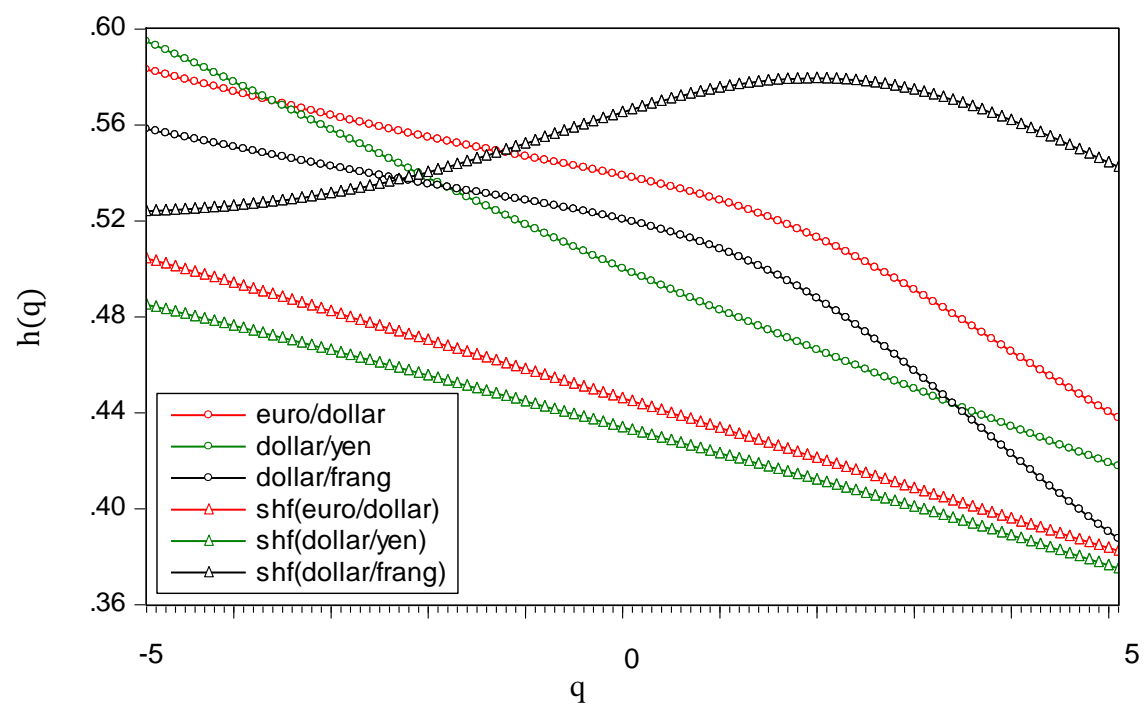

Figure 6. The generalized Hurst exponent h(q) vs. q

Figure 6 was plotted to show the path of the generalized Hurst exponent $h(q)$ versus q order for both the original and shuffled series. According to the results, except for USD/CHF series, the shuffled series still have multifractality features, even though it is lower than the original data. These results exhibit that multifractality of the returns of EUR/USD and USD/JPY arise from the fat tails. In the USD/CHF returns, the slope of the h(q) function has decreased in comparison with the original data. That is, the multifractality of the USD/CHF returns in the shuffled data is quite lower than its original data. However, there are still signs of multifractality in the shuffled USD/CHF returns. This finding demonstrates that the multifractality of the USD/CHF returns stems from both long memory and fat tail features of the series. Table 3 presents the same results in terms of the exact values of the 
generalized Hurst exponents in different q orders. It is clear that the difference between the USD/CHF's generalized Hurst exponent $\mathrm{h}(\mathrm{q})$ values are quite small.

Table 3. The generalized Hurst exponent $\mathrm{h}(\mathrm{q})$ values for different orders

\begin{tabular}{lllllll}
\hline \hline & \multicolumn{3}{c}{ Original Series } & \multicolumn{3}{c}{ Shuffled Series } \\
\hline \hline EUR/USD & $\mathrm{h}(\mathrm{q}=-5)$ & $\mathrm{h}(\mathrm{q}=0)$ & $\mathrm{h}(\mathrm{q}=5)$ & $\mathrm{h}(\mathrm{q}=-5)$ & $\mathrm{h}(\mathrm{q}=0)$ & $\mathrm{h}(\mathrm{q}=5)$ \\
USD/JPY & 0.5830 & 0.5380 & 0.4379 & 0.5043 & 0.4447 & 0.3824 \\
USD/CHF & 0.5949 & 0.4983 & 0.4177 & 0.4850 & 0.4328 & 0.3751 \\
\hline \hline
\end{tabular}

In order to gain a different viewpoint, we presented multifractal spectrum width for the original and shuffled series. As it can be seen from the results, all of the three series' multifractal spectrum widths are more narrow in the shuffled data than the original ones. These results also supported the findings of Figure 6 . If we pay attention, it is clear that the multifractal spectrum width of the EUR/USD and USD/JPY returns in the shuffled series are still high and are bigger than USD/CHF width. The situation shows that although we removed the correlations in the original series, there is still multifractality in the shuffled series of EUR/USD and USD/JPY returns, and it arises from the fat tails.

Table 4. Multifractal Spectrum Width Values

\begin{tabular}{lll}
\hline \hline & Original series & Shuffled series \\
\hline \hline EUR/USD & 0.3108 & 0.2353 \\
USD/JPY & 0.3374 & 0.2184 \\
USD/CHF & 0.3577 & 0.1334 \\
\hline \hline
\end{tabular}

Ihlen (2012) reported that the shape of the multifractal spectrum does not need to be symmetrical. Arising from the negative and positive leveling of the generalized Hurst exponent, respectively, the multifractal spectrum can be left and right truncation. The leveling of the generalized Hurst exponent demonstrates the unsensitivity of the q order root mean square (RMS), versus local fluctuations. If the time series' multifractal structure is unsensitive to small size local fluctuations, the multifractal spectrum will have a long left tail. Otherwise, if the time series has a multifractal structure that is unsensitive to the large size fluctuations, this time the multifractal spectrum will have a long right tail. According to Figure 7, the USD/JPY has a symmetrical multifractal spectrum, whereas the EUR/USD and USD/CHF's multifractal spectrums are in the right truncation shape. This means that the EUR/USD and USD/CHF returns' multifractality is less sensitive to the small size local fluctuations. 


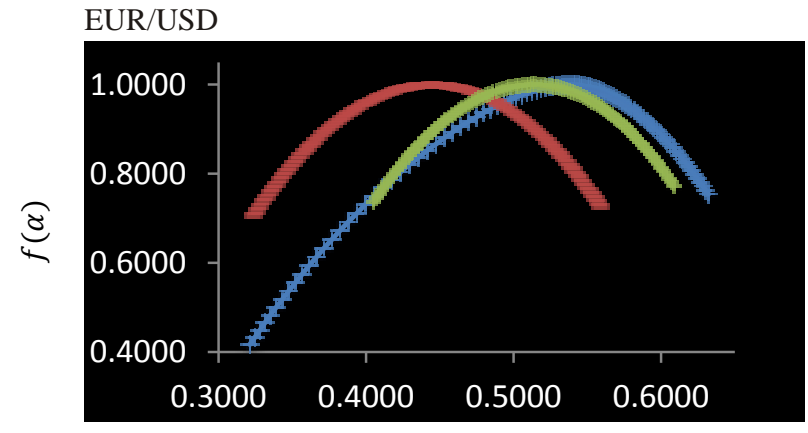

$\alpha$

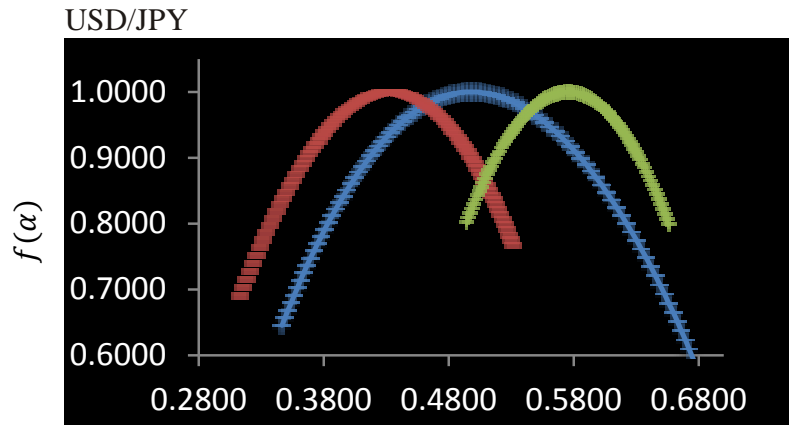

$\alpha$

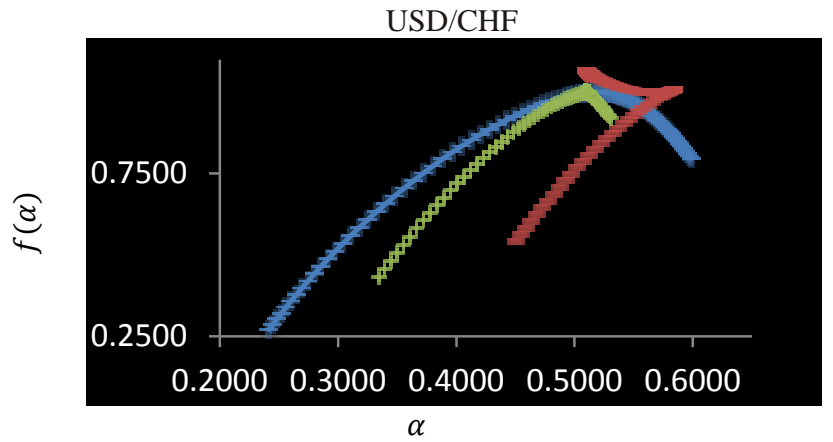

Figure 7. Multifractal Spectrums for Original, Shuffled and Surrogated Series

The second type of multifractality is related to the fat-tails in the probability density function. In order to analyse the existince of the second type, we computed surrogated data via the STAP method and presented the generalized Hurst exponent results of them together with original data results. Table 5 shows that, except for the USD/CHF data, surrogated series' statistics are not quite different from the original data. In spite of that, the gap between the different $\mathrm{q}$ orders for USD/CHF returns in the surrogated data is quite small. Besides, the $\mathrm{h}(\mathrm{q})$ values for the $\mathrm{q}=-5$ and $\mathrm{q}=0$ are almost same. According to the results, there is a fat tails effect in the multifractality of the USD/CHF data besides the long memory.

Table 5. Generalized Hurst Exponents for Original and Surrogated Data

\begin{tabular}{lllllll}
\hline \hline & Original Series & \multicolumn{4}{l}{ Surrogated Series } \\
\hline \hline EUR/USD & $\mathrm{h}(\mathrm{q}=-5)$ & $\mathrm{h}(\mathrm{q}=0)$ & $\mathrm{h}(\mathrm{q}=5)$ & $\mathrm{h}(\mathrm{q}=-5)$ & $\mathrm{h}(\mathrm{q}=0)$ & $\mathrm{h}(\mathrm{q}=5)$ \\
USD/JPY & 0.5830 & 0.5380 & 0.4379 & 0.5633 & 0.5138 & 0.4577 \\
USD/CHF & 0.5949 & 0.4983 & 0.4177 & 0.6164 & 0.5757 & 0.5338 \\
\hline \hline
\end{tabular}

Another finding for the surrogated data is lower multifractalities of the EUR/USD and USD/JPY returns than shuffled data. This situation becomes reversed for the USD/CHF returns, it's multifractality is higher than the shuffled data for the surrogated data. Briefly, we can say that the higher multifractality of the surrogated data compared with the shuffled series substantially arises from the long memory features of the returns, however the higher multifractality of the shuffled series stems from fat-tails. This situation can be seen in Figure 8. In this figure, the $h(q)$ functions of the surrogated and original series were plotted versus q orders. If we pay attention to the USD/CHF returns, we can say that, similar to GBP/USD returns in Figure 3, the slope of the USD/CHF returns' $h(q)$ function seems quite different from the original data. Moreover this slope is almost the same for $\mathrm{q}=-5$ and $\mathrm{q}=0$. This situation shows the effect of the fat-tails in the multifractality of the USD/CHF returns besides the long memory. 


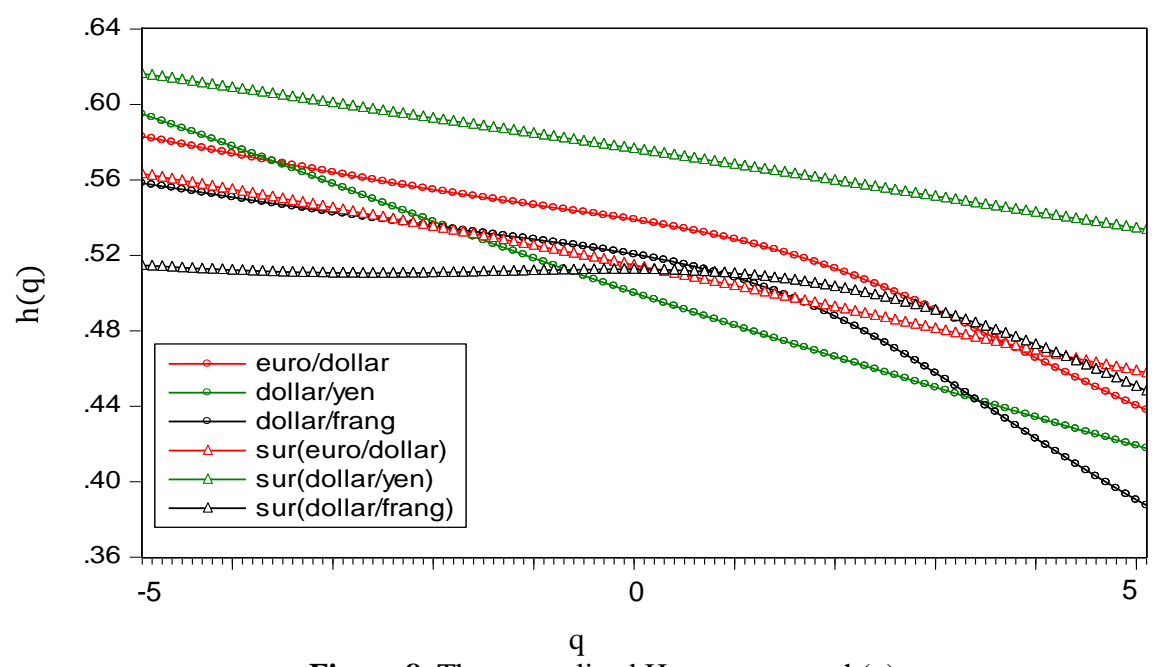

Figure 8. The generalized Hurst exponent h(q) vs. q

While the multifractal structure of the three currencies (EUR/USD, USD/JPY and USD /CHF) arises from the fat tails, we can see that this effect is higher in the EUR/USD and USD/JPY returns. Besides its theoretical meaning, this finding also indicates that individuals and firms that make transactions on these currencies may be exposed to higher risks financially. Because, statistically we know that the factors, which cause these types of fat tails in the return distributions, are instant and extreme events in financial markets. Oil crisis (1973), Asia crisis (1997), Russia crisis (1998) and mortgage crisis (2008) can be given as good examples for these extreme events in the currency markets. In these periods many firms were subjected to large risks in the foreign trade operations. In fact, it can be said that the collapse of LTCM maybe is the best example in this issue. Because, although they used the "modern" mathematical statistics in the risk analysis, as the definition and measurement of the risk and prices processes were wrong, they did not take the extreme events or Black Swans of financial markets into account. On the other hand, in conjunction with the fat tails, another reason of the multifractality, which is long memory, exhibited that return series have a memory property differently from the random walk assumption of the conventional finance theory. This is a very important finding in terms of the market's itself and firms aside from the theoretical approaches. Because in the measurement and hedging of financial risks, today many association still use conventional finance methods that are based on the efficient market hypothesis, random walk and normal distribution assumptions. In every model, which uses normal distribution in the modeling as an assumption, reduces the tail probabilities to insignificant levels. However, our findings demonstrate that fat tails are a very important reason of the multifractal structure of EUR/USD and USD/JPY returns. Therefore it is clear that traditional approaches in the definition and modeling of financial asset returns are not adequate to catch the real characteristics of the prices' process and do not consider stylized facts of these assets. That is why we suggest using Stable Paretian Distributions and multifractal models in order to consider long memory and fat tail features of the financial asset returns. Hurst exponent value of the models that based on the Efficient Market Hypothesis is constant and equal to 0.5. This value means that all these models, such as geometric Brownian motion, take only short memory process into account. However, our findings indicate that there is not only one Hurst exponent value in the returns of EUR/USD, USD/JPY and USD/CHF, that is, these three currency returns exhibit multifractal properties. Nevertheless we know that Black-Scholes option pricing model uses the geometric Brownian motion and this model's Hurst exponent value is 0.5 . Hence, any firm or investor who uses the conventional methods will have some limitations in the modeling and measuring of financial risks. As it is stated before, LTCM is the one of the examples of this reality.

Another thing worth mentioning about the results is liquidity. As we stated, the strongest multifractality features appeared in the USD/EUR and USD/JPY returns. As this situation may be related to the liquidity features of the currency markets, we presented the Bank of International Settlements' (BIS) survey results related to the currency market liquidity in Table 6. 
Table 6. Global Foreign Exchange Market Turnover by Currency Pair ${ }^{1}$

\begin{tabular}{lllllllll}
\hline \hline & 2004 & & 2007 & & 2010 & \multicolumn{2}{c}{2013} \\
\hline \hline Currency Pair & Amount & $\%$ & Amount & $\%$ & Amount & $\%$ & Amount & $\%$ \\
USD / EUR & 541 & 28.0 & 892 & 26.8 & 1.098 & 27.7 & 1.289 & 24.1 \\
USD /JPY & 328 & 17.0 & 438 & 13.2 & 567 & 14.3 & 978 & 18.3 \\
USD /GBP & 259 & 13.4 & 384 & 11.6 & 360 & 9.1 & 472 & 8.8 \\
USD /CHF & 83 & 4.3 & 151 & 4.5 & 166 & 4.2 & 184 & 3.4 \\
\hline \hline
\end{tabular}

Source: https://www.bis.org/publ/rpfx13.htm

According to the information released by the BIS, the USD/EUR and USD/JPY markets are the most liquid currency markets. Although this information seems to support the reason of the highest multifractality of these two markets, we see that the third most liquid market, which is the USD /GBP, does not have multifractality features according to our findings. These results demonstrate that the relationship between liquidity and multifractality is not clear or consistent.

\section{RESULTS}

In this study, we want to analyze the multifractality structure of currency markets. Empirical tests are conducted for the four leading currencies' returns: GBP/USD, EUR/USD, USD/JPY and USD/CHF. All of the data has daily frequency, and the time interval is from 01/01/2004 to 02/25/2014. In the testing of multifractality, we have used the multifractal detrended fluctuation analysis (MF-DFA) developed by Kantelhardt et al. (2002). On the other hand, we have also used surrogated and shuffled data in order to determine the source of multifractality. According to the results, there is no multifractality behavior in the returns of the GBP/USD. All the findings have shown monofractal features for the GBP/USD data. However, we have obtained strong evidence about the multifractality of the EUR/USD, USD/JPY and USD/CHF returns. We have also seen that the reason of the multifractality in the EUR/USD and USD/JPY returns is substantially fat tail, whereas the multifractality of the USD/CHF series arises from both the long memory and fat-tail probability density functions of returns. The last relevant finding is the unclear and inconsistent relationship between multifractality and liquidity of the currency markets.

\section{AUTHOR INFORMATION}

Samet Günay, Ph.D. is an Assistant Professor of Finance in the Department of Banking and Finance, School of Applied Sciences, at Istanbul Arel University, Istanbul, Turkey.

E-mail: dr.sgunay@gmail.com

\section{REFERENCES}

1. Ausloos, M. (2012). Generalized Hurst exponent and multifractal function of original and translated texts mapped into frequency and length time series. Physical Review E, 86 (3), 031108

2. Benbachir, S. \& El Alaoui, M. (2011). A Multifractal Detrended Fluctuation Analysis of the Moroccan Stock Exchange. International Research Journal of Finance and Economics, 78, 6-17

3. Calvet, L. \& Fisher, A. (2002). Multifractality in Asset Returns: Theory and Evidence. The Review of Economics and Statistics, 84 (3), 381-406

4. Caraiani, P. (2012). Evidence of Multifractality from Emerging European Stock Markets. Plosone, 7 (7), 1 9

5. Du, G. \& Ning, X. (2008). Multifractal properties of Chinese stock market in Shanghai. Physica A, 387 (1), 261-269

6. Fillol, J. (2003). Multifractality: Theory and Evidence an Application to the French Stock Market. Economics Bulletin, 3, No. 31 1-12

${ }^{1}$ Daily averages in April, in billions of US dollars and percentages 
7. Hurst, H.E. (1951). Long-term storage capacity of reservoirs. Transactions of the American Society of Civil Engineers, 116, 770-808.

8. Ihlen, E.A.F. (2012). Introduction to multifractal detrended fluctuation analysis in Matlab. Frontiers in Physiology, 3 (141), 1-18

9. Ioan, T., Anita, P. \& Razvan, C. (2012). Multifractal structure of central and eastern European foreign exchange markets. Annals of Faculty of Economics, 1 (1), 784-790.

10. Jiang, Z.Q. \& Zhou, W.X. (2008). Multifractal analysis of Chinese stock volatilities based on partition function approach. Physica A, 387(19), 4881-4888.

11. Kumar, S. \& Deo, N. (2009). Multifractal Properties of the Indian Financial Market. Physica A, 388, 15931602.

12. Kantelhardt, J., S. Zschiegner, E. Koscielny-Bunde, A. Bunde, S. Havlin, and E. Stanley (2002). Multifractal detrended fluctuation analysis of nonstationary time series. Physica A, 316, 87-114

13. Liu R., Di Matteo T. \& Lux T. (2008). Multifractality and Long-Range Dependence of Asset Returns: The Scaling Behaviour of the Markov-Switching Multifractal Model with Lognormal Volatility Components. Kiel Working Paper, 1427, 1-15

14. Lye, C. T. \& Hooy, C. W. (2012). Multifractality and Efficiency: Evidence from Malaysian Sectoral Indices. Int. Journal of Economics and Management, 6 (2), 278 - 294

15. Mandelbrot, B. (1963). The variation of certain speculative prices. The Journal of Business 36(4), 394-419.

16. Mandelbrot, B. (1966). Forecasts of future prices, unbiased markets, and "martingale" models. Journal of Business, 39, 242-255.

17. Mandelbrot, B.B. (1972). Statistical Methodology for Nonperiodic Cycles from Covariance to R/S Analysis. Annals of Economic and Social Measurement, 1, 259-290.

18. Mandelbrot, B. B. (1997). Fractals and Scaling in Finance: Discontinuity, Concentration, Risk, Berlin: Springer

19. Mandelbrot, B.B., Fisher, A. \& Calvet, L. (1997a). A multifractal model of asset returns. Cowles Foundation Discussion Paper, 1164. 1-33

20. Mandelbrot, B. B., Fisher, A. J. \& Calvet, L. E. (1997b). Multifractality of Deutschemark / US Dollar Exchange Rates. Cowles Foundation Discussion Paper, 1166, 1-77

21. Mandelbrot, B. B. \& Hudson, R. L. (2004). The (Mis)behaviour of Markets: A Fractal View of Risk, Ruin, and Reward, Profile Books, London.

22. Movahed, M.S., Jafari, G.R., Ghasemi, F., Rahvar, S. \& Tabar, M.R.R. (2006). Multifractal detrended fluctuation analysis of sunspot time series. Journal of Statistical Mechanics, P02003.

23. Niere, H. M. , (2013). Multifractality in the Philippine Foreign Exchange Market. Management Science and Engineering, 7 (3), 67-70

24. Norouzzadeh, P. \& Rahmani B. (2006). A multifractal detrended fluctuation description of Iranian rial-US dollar exchange rate. Physica A, 367, 328-336.

25. Peng, C. K., Buldyrev S. V., Havlin S., Simons M., Stanley H. E. and Goldberger A. L. (1994). Mosaic organization of DNA nucleotides. Phys. Rev. E, 49 (2), 1685-1689.

26. Peters, E. E. (1994). Fractal Market Analysis: Applying Chaos Theory to Investment and Economics, John Wiley and Sons, Newyork

27. Pleşoianua, A., Todeaa, A. \& Căpuşan, R. (2012). The Informational Efficiency of the Romanian Stock Market: Evidence from Fractal Analysis. Procedia Economics and Finance, 3, 111-118

28. Pochart, B. \& Bouchaud, J.P. (2002). The skewed multifractal random walk with applications to option smiles. Quantitative Finance, 24, 303-314

29. Qian, X. Y., Zhou W. X. \& Gu G. F. (2011). Modified detrended fluctuation analysis based on empirical mode decomposition, Physica A, vol. 390, 23-24, 4388-4395.

30. Segnon, M. \& Lux, T. (2013). Multifractal Models in Finance: Their Origin, Properties, and Applications. Kiel Institute Working Paper, 1860, 1-60

31. Wang, Y., Liu, L. \& Gu, R. (2009). Analysis of Efficiency for Shenzhen Stock Market Based on Multifractal Detrended Fluctuation Analysis. International Review of Financial Analysis, 18, 271-276.

32. Yuan, Y., Zhuang, X.-T., \& Jin, X. (2009). Measuring multifractality of stock price fluctuation using multifractal detrended fluctuation analysis. Physica A, 388(11), 2189- 2197.

33. Zhang L. (2011). Multifractal Properties of the Industry Indices for Chinese and Japanese Stock Markets. International Proceedings of Economics Development \& Research, 12, 497-502 